\title{
Sarcopenia and coronary heart disease synergistically increase the risk of new onset depressive symptoms in older adults
}

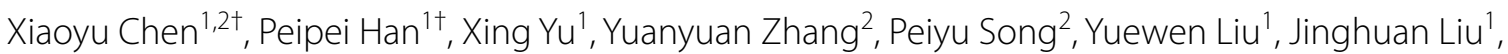 \\ Jiawei Tang ${ }^{1}$, Yisong Zhang ${ }^{1}$, Yong Zhao ${ }^{1}$, Jiejiao Zheng ${ }^{1}$, Lixi Chu ${ }^{1}$, Hong Bing Wang ${ }^{3 *}$ and Qi Guo ${ }^{1 *}$ (D)
}

\begin{abstract}
Background: Coronary heart disease (CHD), sarcopenia and depression are common disorders that markedly impair quality of life and impose a huge financial burden on society. They are also frequently comorbid, exacerbating condition and worsening prognosis. This study aimed to investigate the additive effects of CHD and sarcopenia on the risk of new onset depressive symptoms in older adults.
\end{abstract}

Methods: The prospective cohort study comprised 897 Chinese community-dwelling participants who were aged 60 years and older (386 men; mean age 66.9 \pm 5.9years) without depressive symptoms at baseline, recruited from Chadian of Tianjin, China. Sarcopenia was defined according to the Asian Working Group for Sarcopenia (AWGS) criteria. CHD was identified via medical records or new diagnosed by at least two physicians. Depressive symptoms were assessed using the Geriatric Depression Scale (GDS) $\geq 11$. Longitudinal data on new onset depressive symptoms were collected up to 12 months after baseline.

Results: We found that 103 (11.5\%) of the 897 participants without depressive symptoms at baseline had developed depressive symptoms. Participants were classified into mutually exclusive groups based on sarcopenia status and CHD: normal, CHD alone, sarcopenia alone, and co-occurring groups. A logistic regression showed that the CHD alone [odd ratios $(\mathrm{OR})=1.78,95 \%$ confidence interval $(\mathrm{Cl})=1.05-3.02$ ], sarcopenia alone $(\mathrm{OR}=2.79,95 \% \mathrm{Cl}=1.26-6.22)$, and co-occurring $(\mathrm{OR}=7.19,95 \% \mathrm{Cl}=2.75-18.81)$ had higher risk of depressive symptoms than the normal group after adjusting for the covariates.

Conclusions: CHD and sarcopenia synergistically increase the risk of new onset depressive symptoms in older adults. Thus, older adults may require early detection, and appropriate interventions should be implemented.

Keywords: Coronary heart disease, Depressive symptoms, Elderly, Sarcopenia

\footnotetext{
*Correspondence: 15301799929@163.com; guoqijp@gmail.com

${ }^{\dagger}$ Xiaoyu Chen and Peipei Han contributed equally to this work and should be considered co-first author.

${ }^{1}$ Department of Rehabilitation Medicine, Shanghai University of Medicine and Health Sciences Affiliated Zhoupu Hospital, 1500 Zhouyuan Road, Pudong New District, Shanghai 201318, China

${ }^{3}$ Department of Rehabilitation Medicine, Shanghai Fourth Rehabilitation Hospital, Shanghai 200040, China

Full list of author information is available at the end of the article
}

\section{Background}

Depression is one of the most significant mental disorders associated with later life. A previous study reported that the prevalence of depressive symptoms among community-dwelling older adults varies from 8 to $16 \%$ [1]. Depression is a major risk factor for suicide, and is also related to considerable morbidity and mortality [2, 3]. Due to the severe side effects associated with depressive symptoms, researchers have sought to identify the 
risk factors for depression in elderly individuals and aim to reduce the incidence of depressive symptoms through intervention. Growing evidence supports that the circulatory and muscular systems are known to be associated with depression $[4,5]$. Therefore, psychological and physical indicators need to be integrated together, so as to prevent the occurrence and development of diseases.

Coronary heart disease (CHD) is a psychosomatic disease with a prevalence of $19.3 \%$ in the older adults [6]. $\mathrm{CHD}$ and depression are also frequently comorbid, exacerbating the patient conditions and worsening prognosis. A recent review has reported that approximately $40 \%$ of people with CHD will also suffer from some form of depression, and as such are an important patient group, because they have worse physical health outcomes associated with CHD compared to similar patients without depression [7]. Meanwhile, accumulating evidence indicates that the onset of the episodes of depressive symptoms may be important in predicting worse outcomes among people with CHD [7, 8]. Accordingly, it should be expected that people with CHD at higher risk of newonset depression would benefit from detection in enough time to prevent adverse outcomes. In addition, the direction of association between CHD and depressive symptoms is unclear, which needs further research. Therefore, a better understanding of comorbid CHD and depressive symptoms is particularly important for improving CHD management in older adults and thus achieving a healthier aging society.

It is noteworthy that sarcopenia is common in the elderly, with a prevalence in our previous study of nearly $10 \%$ [9]. Sarcopenia, defined as age-related loss of muscle mass and function [10], is a morbid condition in older adults that can result in serious health consequences, such as falls, disability and mortality [11]. Although the causal association between sarcopenia and depressive symptoms is still unclear, our previous cohort study showed that sarcopenia was an independent risk factor of depressive symptoms in suburb-dwelling older adults [12]. Furthermore, previous studies revealed that there may be an interaction relationship between sarcopenia and CHD [13-15]. CHD and sarcopenia often affect the same elderly individuals, seriously affecting the health and quality of life of the older adults. Thus, we have reason to believe that $\mathrm{CHD}$ and sarcopenia might be associated, accelerating the negative cycle of depressive symptoms through these interaction pathways. The high prevalence of these two factors makes the study of their joint effect very valuable. Inactivity may lead to $\mathrm{CHD}$, meaning the physical decline of patients with CHD should be focused on to avoid the appearance of sarcopenia. When one risk has already emerged, we can control the other to prevent further complications. However, combinatory effects of these two conditions on depressive symptoms still remains unclear. Therefore, the aim of this study was to examine whether CHD and sarcopenia were independent risk factors, and whether they synergistically lead to an increase in new-onset depressive symptoms in Chinese community-dwelling older adults, using longitudinal cohort data.

\section{Methods \\ Study participants}

Our study population consisted of residents of the Hangu area of Tianjin, China, who were aged $\geq 60$ years old and participated in China's national free physical examination program. Participants gave full, informed written consent to take part in the study and ethical approval was approved by the Ethics Committee at our University, and the methods were carried out in accordance with the principles of the Declaration of Helsinki. In this study analysis, data collected in July 2015 and 2016 were used as the baseline, and new cases of depressive symptoms were follow-up in July 2016 and 2017.

The inclusion criteria were volunteer to join in the study and absence of depressive symptoms at baseline. Exclusion criteria were as follows: (1) did not complete the assessment of depressive symptoms; (2) did not have the date of CHD diagnosis; (3) lack of relevant data for the assessment of sarcopenia or (4) cannot talk to interviewers or to grant informed consent. Baseline variable data were available for 1045 participants, although 80 older adults with depressive symptoms were excluded at baseline, 24 individuals were lost to follow-up (5 died, 7 institutionalized, 12 bedridden), and a further 44 had missing information for covariates or outcomes. Therefore, the final analytic date consisted of 897 participants (Fig. 1).

\section{CHD definitions}

CHD history was obtained via medical records and reviewed by a cardiologist and a general practitioner, which included acute myocardial infarction, angina pectoris, percutaneous coronary intervention (PCI) or coronary artery bypass graft (CABG), and all other ischemic heart disease. In undiagnosed participants, at least two physician claims with a CHD diagnosis could be considered CHD [16].

\section{Assessment of sarcopenia and depressive symptoms}

Sarcopenia was defined using the diagnostic algorithm according to the AWGS criteria [10] and the 30-item Geriatric Depression Scale (GDS) [17] was administered by conducting an interview to assess depressive symptoms [18]. Details of measurement methods can be found in our previous study [12]. 


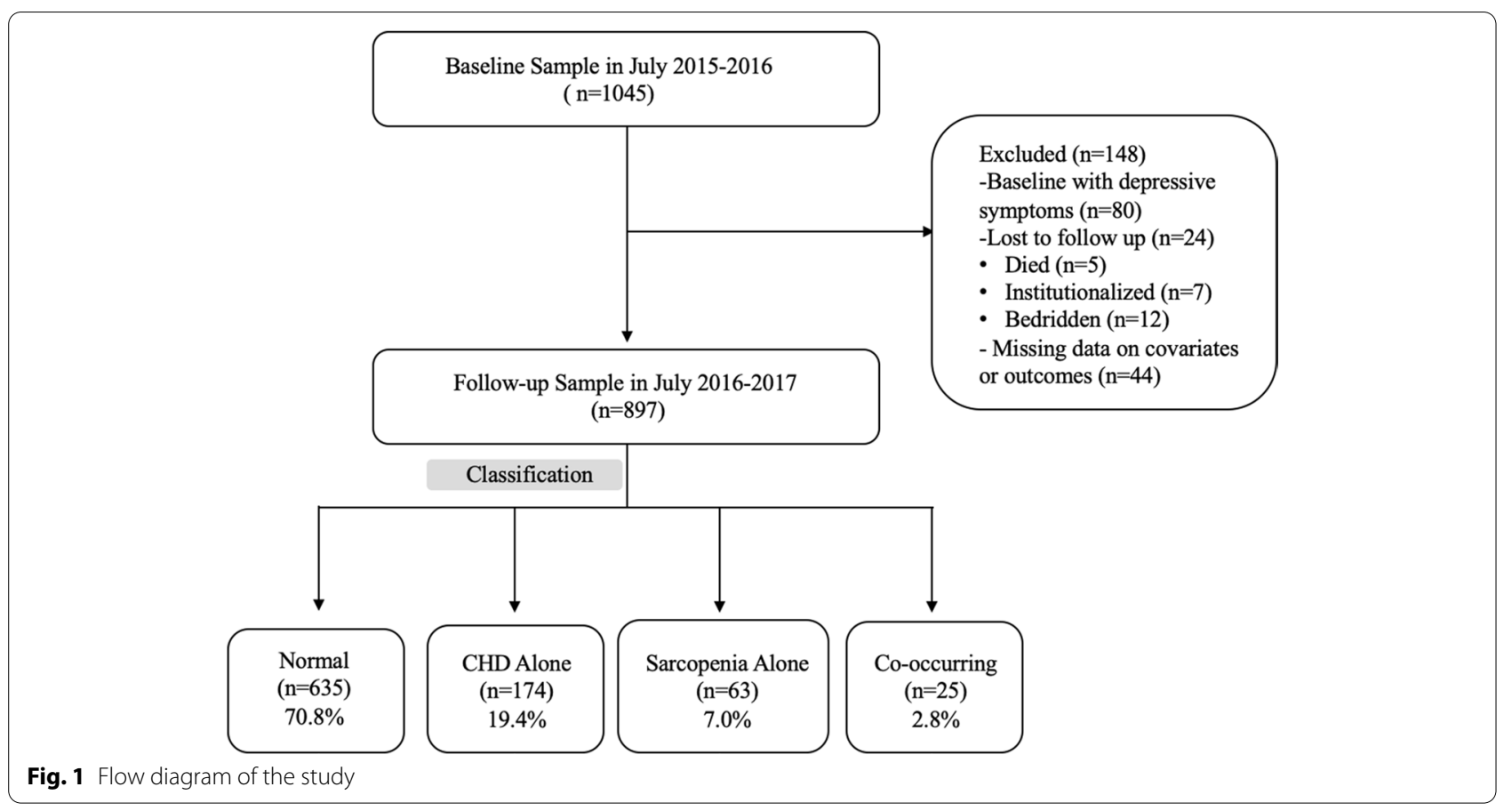

\section{Covariates}

Data related to sociodemographic variables (age, gender, marital status, educational level and occupation) and behavioral characteristics (smoking and drinking habits, sleep behavior, and history of falls). The short form of the International Physical Activity Questionnaire (IPAQ) [19] was used to evaluate the physical activity. We used the Mini Nutritional Assessment-Short Form (MNA-SF) [20], a validated screening tool used in geriatric health care, to evaluate the nutritional status, which had high sensitivity, specificity and correlation to the full MNA [21]. Comorbidity was assessed using the Charlson Comorbidity Index (CCI) [22] and current use of drugs included hypotensive drugs, hypoglycemic drugs, lipid-lowering drugs, cardiovascular drugs, psychotropic drugs, gastrointestinal drugs, or sleep drugs. Details of measurement methods have been described in our previous study [12] [23].

\section{Statistics}

Participants were classified into mutually exclusive groups based on sarcopenia status and CHD status: normal, CHD alone, sarcopenia alone, and co-occurring. The study participants' characteristics were compared by sarcopenia and CHD status using Student t-test or the Manne-Whitney U-test for continuous variables and the chi-square test for categorical variables. Using logistic regression analyses, we initially estimated odds ratio (OR) and 95\% confidence intervals (CI) of sarcopenia alone, CHD alone, and both to assess the additive effects of sarcopenia and CHD on new onset depressive symptoms. Participants with no sarcopenia and no CHD were categorized as the reference group. The final model adjusted for age, sex, BMI, widowed, education, falling history, drinking, MNA-SF, sleep duration, sleep quality, hypotensive drugs, hypoglycemic drugs, cardiovascular drugs, sleep drugs and CCI. All statistical analyses were performed using SPSS v 25.0 (SPSS Inc., Chicago, IL), and $P$ values of less than 0.05 were considered statistically significant.

\section{Results}

Figure 1 shows the participants over the follow-up period. The analytic sample comprised 897 study participants (386 men; mean age at baseline $66.9 \pm 5.9$ years). At 1-year follow-up, there were 103 (11.5\%) people with new onset elevated depressive symptoms. Demographics and clinical characteristics of the 4 groups are presented in Table 1.

Results of the new onset depressive symptoms and logistic regression analysis using the 4 groups are shown in Table 2. New onset depressive symptoms rates of the 4 groups were $8.2 \%(n=52), 16.7 \%(n=29), 19.0 \%$ $(n=12)$, and $40.0 \%(n=10)$ in normal, CHD alone, sarcopenia alone, and co-occurring groups, respectively. Individuals with the new onset depressive symptoms in CHD or sarcopenia group were significantly more than normal group, whereas the co-occurring group 
Table 1 Baseline Characteristics of Study Participants absence of Depressive Symptoms at baseline

\begin{tabular}{|c|c|c|c|c|c|}
\hline Variables & $\begin{array}{l}\text { Normal } \\
(n=635)\end{array}$ & $\begin{array}{l}\text { CHD Alone } \\
(n=174)\end{array}$ & $\begin{array}{l}\text { Sarcopenia Alone } \\
(n=63)\end{array}$ & $\begin{array}{l}\text { Co-occurring } \\
(n=25)\end{array}$ & $P$-value \\
\hline Age (y) & $66.0 \pm 5.5$ & $67.3 \pm 5.6^{a}$ & $72.4 \pm 6.5^{a, b}$ & $71.4 \pm 6.3^{a, b}$ & $<0.001$ \\
\hline Sex (Male) & $314(49.4)$ & $48(27.6)^{\mathrm{a}}$ & $19(30.2)^{a}$ & $5(20.0)^{a}$ & $<0.001$ \\
\hline BMI $\left(\mathrm{kg} / \mathrm{m}^{2}\right)$ & $25.39 \pm 3.30$ & $26.02 \pm 3.64^{\mathrm{a}}$ & $22.68 \pm 3.11^{a, b}$ & $22.51 \pm 2.71^{a, b}$ & $<0.001$ \\
\hline Grip strength (kg) & $26.51 \pm 9.53$ & $23.32 \pm 9.25^{\mathrm{a}}$ & $16.04 \pm 6.32^{a, b}$ & $16.52 \pm 8.03^{a, b}$ & $<0.001$ \\
\hline $\mathrm{SMI}\left(\mathrm{kg} / \mathrm{m}^{2}\right)$ & $7.31 \pm 1.01$ & $7.08 \pm 1.09$ & $5.43 \pm 0.91^{a, b}$ & $5.43 \pm 0.56^{a, b}$ & $<0.001$ \\
\hline Gait speed (m/s) & $1.01 \pm 0.17$ & $0.96 \pm 0.16^{a}$ & $0.85 \pm 0.19^{a, b}$ & $0.77 \pm 0.22^{a, b}$ & $<0.001$ \\
\hline IPAQ (Met/wk) & $2142(924,4147)$ & $1848(693,4186)$ & $2079(1386,3066)$ & $1038(0,4697)$ & 0.732 \\
\hline Widowed (\%) & $76(12.0)$ & $26(14.9)^{\mathrm{a}}$ & $21(33.3)^{b}$ & $6(24.0)$ & 0.001 \\
\hline Living alone (\%) & $90(14.2)$ & $21(12.1)$ & $16(25.4)$ & $5(20.0)$ & 0.253 \\
\hline Illiteracy (\%) & $152(24.0)$ & $45(25.9)$ & $28(44.4)^{\mathrm{a}}$ & $7(28.0)$ & 0.006 \\
\hline Farming (\%) & $559(88.2)$ & 156(89.7) & $57(90.5)$ & $22(88.0)$ & 0.911 \\
\hline Fall history (\%) & $84(13.2)$ & $25(14.4)$ & $12(19.0)$ & $8(32.0)$ & 0.044 \\
\hline Drinking (\%) & $90(14.2)$ & $12(6.9)^{\mathrm{a}}$ & $8(12.7)$ & $0(0.0)$ & 0.002 \\
\hline Smoking (\%) & $180(28.4)$ & $47(27.2)$ & $22(34.9)$ & $10(40.0)$ & 0.326 \\
\hline MNA-SF & $13.06 \pm 1.12$ & $13.15 \pm 0.90$ & $12.19 \pm 1.74^{a, b}$ & $12.08 \pm 1.58^{\mathrm{a}, \mathrm{b}}$ & $<0.001$ \\
\hline Sleep Duration (h) & $7.87 \pm 1.32$ & $8.12 \pm 1.47$ & $8.39 \pm 1.30^{\mathrm{a}}$ & $8.36 \pm 2.33$ & 0.005 \\
\hline Sleep quality (\%) & & & & & 0.003 \\
\hline Very well & $339(53.4)$ & $65(37.6)^{\mathrm{a}}$ & $29(46.0)$ & $7(28.0)$ & \\
\hline Good & 186(29.3) & $61(35.3)$ & 20(31.7) & $9(36.0)$ & \\
\hline Not enough & $56(8.5)$ & 20(11.6) & $6(9.5)$ & $6(24.0)$ & \\
\hline Very poor & $54(8.5)$ & $27(15.6)$ & $8(12.7)$ & $3(12.0)$ & \\
\hline \multicolumn{6}{|l|}{ Medicine use (\%) } \\
\hline Hypotensive drugs & $222(35.0)$ & $88(50.6)^{\mathrm{a}}$ & $21(33.3)^{b}$ & $10(40.0)$ & $<0.001$ \\
\hline Hypoglycemic drugs & $41(6.5)$ & $27(15.5)^{\mathrm{a}}$ & $4(6.3)^{b}$ & $3(12.0)$ & $<0.001$ \\
\hline Lipid-lowering drugs & $78(12.3)$ & 19(10.9) & $2(3.2)$ & $3(12.0)$ & 0.237 \\
\hline Cardiovascular drugs & $2(0.3)$ & $102(58.6)^{\mathrm{a}}$ & $3(4.8)^{b}$ & $11(44.0)^{\mathrm{a}, \mathrm{c}}$ & $<0.001$ \\
\hline Gastrointestinal drugs & $16(2.5)$ & $3(1.7)$ & $3(4.8)$ & $1(4.0)$ & 0.382 \\
\hline Sleep drugs & $34(5.4)$ & $24(13.8)^{\mathrm{a}}$ & $7(11.1)$ & $5(20.0)^{\mathrm{a}}$ & $<0.001$ \\
\hline \multicolumn{6}{|l|}{ Chronic conditions (\%) } \\
\hline Diabetes & $66(10.4)$ & $30(17.2)$ & $14(22.2)$ & $6(24.0)^{a, b}$ & 0.003 \\
\hline Hypertension & $320(50.4)$ & $116(66.7)^{a}$ & $29(46.0)^{b}$ & $17(68.0)$ & $<0.001$ \\
\hline Hyperlipidemia & $236(37.2)$ & $80(46.0)$ & $11(17.5)^{a, b}$ & $11(44.0)$ & 0.001 \\
\hline Stroke & $36(5.7)$ & $15(8.6)$ & $6(9.5)$ & $3(12.0)$ & 0.257 \\
\hline Kidney disease & $24(3.8)$ & $10(5.7)$ & $2(3.2)$ & $1(4.0)$ & 0.684 \\
\hline Hepatic disease & $11(1.7)$ & $2(1.1)$ & $\mathrm{O}(0.0)$ & $2(8.0)$ & 0.059 \\
\hline Biliary tract disease & $19(3.0)$ & $10(5.7)$ & $4(6.3)$ & $2(8.0)$ & 0.164 \\
\hline Peptic ulcer & $22(3.5)$ & $12(6.9)$ & $4(6.3)$ & $2(8.0)$ & 0.160 \\
\hline Osteoarthritis & $82(12.9)$ & 33(19.0) & $8(12.7)$ & $4(16.0)$ & 0.231 \\
\hline Parkinson disease & $2(0.3)$ & $1(0.6)$ & $0(0.0)$ & $1(4.0)$ & 0.316 \\
\hline Gout & $3(0.5)$ & $2(1.1)$ & $0(0.0)$ & $0(0.0)$ & 0.642 \\
\hline $\mathrm{CCl}$ & $2.45 \pm 0.76$ & $3.63 \pm 0.76^{a}$ & $3.24 \pm 0.88^{a, b}$ & $4.32 \pm 1.31^{a, b, c}$ & $<0.001$ \\
\hline
\end{tabular}

Notes: $C H D$ coronary heart disease; BMI body mass index; SMI skeletal muscle index; IPAQ international physical activity questionnaire; MET/wk metabolic equivalent task minutes per week; MNA-SF Mini Nutritional Assessment-Short Form; CCI Charlson Comorbidity Index

a $P<0.05$ versus Normal, ${ }^{b} P<0.05$ versus CHD alone, ${ }^{c} P<0.05$ versus Sarcopenia alone

owned significantly most elderly with new onset depressive symptoms $(P<0.05)$. After adjustments for potential confounders, the following groups were associated with depressive symptoms incidence from baseline to 1-year follow up, respectively: $\mathrm{CHD}$ alone $(\mathrm{OR}=1.78$, $95 \% \mathrm{CI}=1.05-3.02)$, sarcopenia alone $(\mathrm{OR}=2.79$, 
Table 2 Association of Co-occurring Sarcopenia and CHD with New onset of Depressive Symptoms

\begin{tabular}{|c|c|c|c|c|c|}
\hline Variables & Normal & CHD alone & Sarcopenia alone & Co-occurring & $P$-value \\
\hline $\begin{array}{l}\text { The onset depressive } \\
\text { symptoms (\%) }\end{array}$ & $52(8.2)$ & $29(16.7)^{\mathrm{a}}$ & $12(19.0)^{\mathrm{a}}$ & $10(40.0)^{a, b, c}$ & $<0.001$ \\
\hline \multicolumn{6}{|c|}{ Logistic regression analyses odd ratio (95\% Cl) } \\
\hline Unadjusted & 1.00(Reference) & $2.24(1.38,3.66)^{\mathrm{a}}$ & $2.64(1.32,5.26)^{\mathrm{a}}$ & $7.47(3.20,17.47)^{a, b, c}$ & \\
\hline Adjusted $^{1}$ & 1.00 (Reference) & $1.78(1.05,3.02)^{\mathrm{a}}$ & $2.79(1.26,6.22)^{\mathrm{a}}$ & $7.19(2.75,18.81)^{a, b, c}$ & \\
\hline
\end{tabular}

Notes: $\mathrm{Cl}$ confidence interval

1 Adjusted for age, sex, BMI, widowed, education, falling history, drinking, MNA-SF, sleep duration, sleep quality, hypotensive drugs, hypoglycemic drugs, cardiovascular drugs, sleep drugs and $\mathrm{CCl}$

a $P<0.05$ versus Normal, ${ }^{b} P<0.05$ versus CHD alone, ${ }^{c} P<0.05$ versus Sarcopenia alone

95\% $\mathrm{CI}=1.26,6.22)$, and co-occurring $(\mathrm{OR}=7.19,95 \%$ $\mathrm{CI}=2.75-18.81)$ groups. No statistical difference was found between CHD alone individuals and sarcopenia alone individuals. However, a significant difference between co-occurring group with $\mathrm{CHD}$ alone or sarcopenia alone group or the normal group $(P<0.05)$ (Fig. 2).

\section{Discussion}

To the best of our knowledge, this is the first prospective study to clarify the combined effects of CHD and sarcopenia on new-onset depressive symptoms. In addition, after adjusting for potential confounders, CHD and sarcopenia were found to be independent risk factors of new-onset depressive symptoms. Co-occurring CHD and sarcopenia presented the highest risk of incidence of depressive symptoms in the study population.

In our study, the association between sarcopenia and depression has been discussed deeply in our previous studies [12]. The most compelling finding was that sarcopenia combined with CHD had a further additive predictive value in discriminating older adults at high risk of new-onset depressive symptoms. Previous studies have reported that sarcopenia is closely related to a higher prevalence of established CHD. The presence of sarcopenia is also a contributing factor in poor cardiopulmonary function in patients with CHD [13-15]. Kim et al.

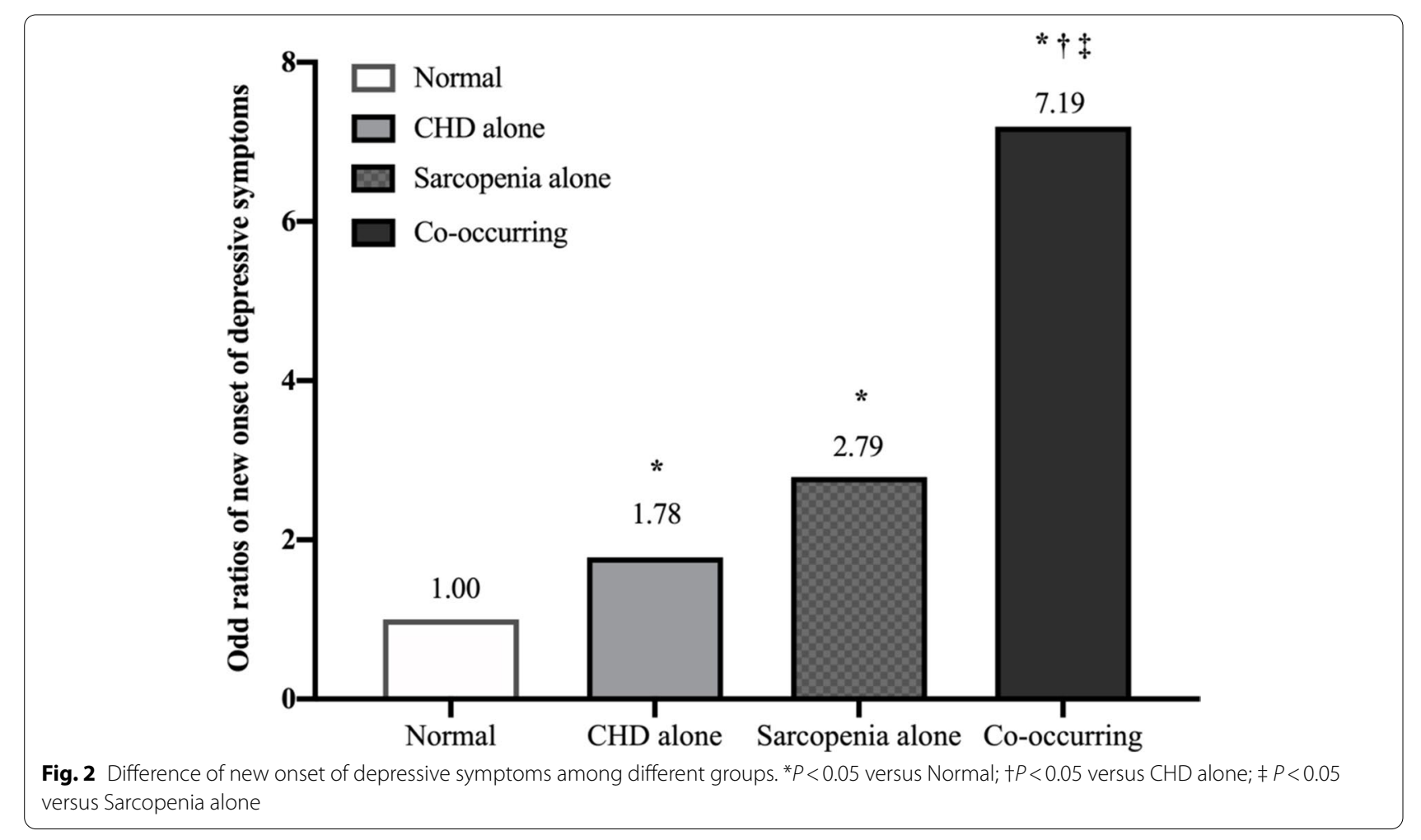


[24] believe that the existence of sarcopenia increases the morbidity and mortality of cardiovascular diseases in the elderly. In patients with sarcopenia, the endocrine function of muscle cells is weakened, and muscle cells exert their endocrine functions by secreting cytokines that are beneficial to the cardiovascular system. Both a decrease in the amount of muscle cells and a decline in their endocrine function in patients with sarcopenia could have contributed to the poor clinical outcomes [25]. Behavioural, genetic and inflammatory mechanisms, as well as changes in hormone levels, decreased physical activity, insulin resistance that could explain the presence of sarcopenia and coronary heart disease are linked to depressive symptoms. On the other hand, polyunsaturated omega-3 free fatty acid deficiency, hypothalamic-pituitary-adrenal axis and autonomic mechanisms are also possible link pathways [26].

Another possible underlying mechanism to explain our results is that sarcopenia and CHD form a vicious cycle. Previous research suggested that low muscle mass and muscle strength are associated with risk of atherosclerosis and endothelial dysfunction in the elderly [13, 27], which may worsen the progression of CHD. Our additional results demonstrated that poor physical performance (gait speed $<0.8 \mathrm{~m} / \mathrm{s}$ was associated with new onset of CHD (adjusted $\mathrm{OR}=2.08,95 \% \mathrm{CI}=1.08-4.00$ ). At the same time, CHD can lead to a decrease in gait speed, reducing people's willingness to attempt tasks that they are otherwise capable of performing [28]. Subsequently, this decrease in physical performance and increase in restriction might lead to decline of muscle mass or strength, eventually leading to sarcopenia. Based on these previously discussed studies and our current findings, sarcopenia and CHD in older adults appears to have an interaction effect on new-onset depressive symptoms. However, the specific mechanism is still unclear and needs to be confirmed further studies involving larger populations and with longer follow-up durations.

Major differences in body composition, risk of CHD and risk of depression are known to exist between the sexes. In this study, however, we did not explore stratification by gender because of our small sample size. If stratified by gender, co-occurring groups were associated with depressive symptoms incidence from baseline to 1-year follow up in both men and women (men: $\mathrm{OR}=18.58,95 \% \mathrm{CI}=2.26-152.65$; women: $\mathrm{OR}=8.51$, $95 \% \mathrm{CI}=2.61-27.74)$. The man group had a larger confidence interval because there were fewer people in the co-occurring group $(n=5)$, which would limit the reliability of the statistical analysis. However, we can find that the results are statistically significant for both men and women, and the deficiency is that the sample size is small. Therefore, in our statistical model, the final results were adjusted for gender and not stratified by sex in the study. This is consistent with several previous studies, which explored the sarcopenia with depression or CHD without grouping studies by gender [12]. In the future, we need to further expand the sample size to explore whether there are gender differences between sarcopenia, $\mathrm{CHD}$ and depression.

This study suggests that older adults with CHD and sarcopenia should be identified early and targeted, so that further mental deterioration and other adverse health outcomes can be prevented. In particular, the American Heart Association (AHA) showed that depression was a risk factor for adverse medical outcomes in patients with CHD [29]. Through this research, we have found the need to focus on physical function. In addition to exercise and nutrition to improve physical function and relieve depression, recent study shows that new drugs can significantly improve depressive symptoms in patients with heart disease [30]. Therefore, much greater attention must be paid to mental health during cardiac rehabilitation and multifaceted interventions require to be considered.

\section{Strengths and limitations}

The strength of this study is that it is the first study to report that CHD with sarcopenia is a stronger risk factor for incidence of depressive symptoms. Moreover, findings may provide new insights for cardiac rehabilitation in older adults. Despite extensive efforts to curb study limitations, some limitations did still exist. First, the present study didn't describe the severity of CHD or its treatment in sarcopenia patients with CHD. In addition, our participants were relatively healthy, we didn't include participants who were unable to participate in the free annual national physical examination (eg, those bedridden or with serious disease). Given these reasons, the population studied may not be comprehensive enough. As a result, we may have underestimated the prevalence of sarcopenia, CHD or depressive symptoms. Thirdly, sarcopenia is a major risk factor for frailty, and frailty also has links with depressive symptoms and CHD. We need to acknowledge that the failure to assess frailty is a limitation of this study and we may pay more attention to the older adults with frailty in the future. Fourthly, this study sample was enrolled from a free physical examination program, and the sample size is small, rather than a representative sample. Sarcopenic obesity may act together to increase their effect on metabolic disorders, cardiovascular and mortality [31], while the small number of sample limited our research on sarcopenic obesity. Furthermore, the sample sizes limited our exploration of gender stratification. In the future, when our sample size 
expands, we will further stratify by gender to explore the correlation between sarcopenic obesity, CHD and depression. Lastly, the follow-up period was short. In future research, we plan to enlarge sample sizes and extend the time for follow-up, to increase the ability to evaluate relationships.

\section{Conclusions}

In conclusion, we have showed the distinct and synergistic effects of sarcopenia and CHD on the risk of newonset depressive symptoms among community-dwelling older adults in a longitudinal study design, after adjusting for confounding factors. To manage and prevent poor outcomes in older adults, physical, nutritional and psychological assessment and interventions should be implemented to allow patients to maintain functional independence and extend healthy life span expectancy.

\begin{abstract}
Abbreviations
CHD: Coronary heart disease; BMI: Body mass index; SMI: Skeletal muscle index; AWGS: Working Group for Sarcopenia; GDS: Geriatric Depression Scale; MNA-SF: Mini Nutritional Assessment-Short Form; IPAQ: International Physical Activity Questionnaire; MET/wk.: Metabolic equivalent task minutes per week; CCl: Charlson Comorbidity Index; PCl: Percutaneous coronary intervention; CABG: Coronary artery bypass graft; AHA: American Heart Association.
\end{abstract}

\section{Acknowledgements}

The authors thank Guiyan shao from the Chadian public health center and Xiaofang Ren from Hangu welfare house for providing the place and organization.

\section{Authors' contributions}

Study concept and design: XC, PH and QG; Acquisition, analysis and interpretation of data: $X Y, Y Z 1, P S, Y L$, JL, JT and YZZ; Drafting of the work: $X C$ and $\mathrm{PH}$; Critical revision of the manuscript: $X C, Y Z 3, J Z, L C, H W$ and QG. All authors have read and approved the manuscript.

\section{Funding}

This work was supported by the funding of the National Natural Science Foundation of China (82172552); Youth Fund Project of Research Planning Foundation on Humanities and Social Sciences of the Ministry of Education (20YJCZH001); Shanghai Sailing Program(20YF1418200). The funding bodies had an important role in the design of the study; collection, analysis, and interpretation of data and writing of the manuscript.

\section{Availability of data and materials}

The datasets used and/or analyzed during the current study are available from the corresponding author on reasonable request.

\section{Declarations}

\section{Consent to publication}

Not Applicable.

\section{Ethics approval and consent to participate}

The study procedures were carried out by the Declaration of Helsinki. The Institutional Review Board of the Ethics Committee at Tian Medical University approved this study. All participants were informed about the study, and all provided written informed consent.

\section{Competing interests}

The authors declare that they have no conflict of interest.

\section{Author details}

'Department of Rehabilitation Medicine, Shanghai University of Medicine and Health Sciences Affiliated Zhoupu Hospital, 1500 Zhouyuan Road, Pudong New District, Shanghai 201318, China. ${ }^{2}$ Department of Rehabilitation Medicine, Tianjin Medical University, Tianjin 300070, China. ${ }^{3}$ Department of Rehabilitation Medicine, Shanghai Fourth Rehabilitation Hospital, Shanghai 200040, China.

Received: 18 November 2020 Accepted: 10 December 2021

Published online: 24 December 2021

\section{References}

1. Blazer DG. Depression in late life: review and commentary. J Gerontol A Biol Sci Med Sci. 2003;58(3):249-65.

2. Alexopoulos GS. Depression in the elderly. Lancet. 2005;365(9475):1961-70.

3. Eurelings LS, Van Dalen JW, Ter Riet G, Moll van Charante EP, Richard E, van Gool WA, et al. Apathy and depressive symptoms in older people and incident myocardial infarction, stroke, and mortality: a systematic review and meta-analysis of individual participant data. Clin Epidemiol. 2018;10:363-79.

4. Piwonski J, Piwonska A, Jedrusik P, Stokwiszewski J, Rutkowski M, Bandosz P, et al. Depressive symptoms and cardiovascular diseases in the adult polish population. Results of the NATPOL2011 study. Kardiol Pol. 2019;77(1):18-23

5. Heo JE, Shim JS, Song BM, Bae HY, Lee HJ, Lee E, et al. Association between appendicular skeletal muscle mass and depressive symptoms: review of the cardiovascular and metabolic diseases etiology research center cohort. J Affect Disord. 2018;238:8-15.

6. Zhu KF, Wang YM, Zhu JZ, Zhou QY, Wang NF. National prevalence of coronary heart disease and its relationship with human development index: a systematic review. Eur J Prev Cardiol. 2016;23(5):530-43.

7. Dickens C. Depression in people with coronary heart disease: prognostic significance and mechanisms. Curr Cardiol Rep. 2015;17(10):83.

8. Carney RM, Freedland KE. Depression and coronary heart disease. Nat Rev Cardiol. 2017;14(3):145-55.

9. Han P, Kang L, Guo Q, Wang J, Zhang W, Shen S, et al. Prevalence and factors associated with sarcopenia in suburb-dwelling older Chinese using the Asian working Group for Sarcopenia Definition. J Gerontol A Biol Sci Med Sci. 2016;71(4):529-35.

10. Chen LK, Liu LK, Woo J, Assantachai P, Auyeung TW, Bahyah KS, et al. Sarcopenia in Asia: consensus report of the Asian working Group for Sarcopenia. J Am Med Dir Assoc. 2014;15(2):95-101.

11. Beaudart C, Zaaria M, Pasleau F, Reginster JY, Bruyere O. Health outcomes of sarcopenia: a systematic review and Meta-analysis. PLoS One. 2017;12(1):e0169548.

12. Chen X, Guo J, Han P, Fu L, Jia L, Yu H, et al. Twelve-month incidence of depressive symptoms in suburb-dwelling Chinese older adults: role of sarcopenia. J Am Med Dir Assoc. 2019;20(1):64-9.

13. Campos AM, Moura FA, Santos SN, Freitas WM, Sposito AC. Brasilia Study on Healthy A, Brasilia Heart S. Sarcopenia, but not excess weight or increased caloric intake, is associated with coronary subclinical atherosclerosis in the very elderly. Atherosclerosis. 2017;258:138-44.

14. Nichols S, O'Doherty AF, Taylor C, Clark AL, Carroll S, Ingle L. Low skeletal muscle mass is associated with low aerobic capacity and increased mortality risk in patients with coronary heart disease - a CARE CR study. Clin Physiol Funct Imaging. 2019;39(1):93-102.

15. Xu L, Hao YT. Effect of handgrip on coronary artery disease and myocardial infarction: a Mendelian randomization study. Sci Rep. 2017;7(1):954.

16. Anderson JL, Adams CD, Antman EM, Bridges CR, Califf RM, Casey DE Jr, et al. 2011 ACCF/AHA focused update incorporated into the ACC/AHA 2007 guidelines for the Management of Patients with Unstable Angina/ non-ST-elevation myocardial infarction: a report of the American College of Cardiology Foundation/American Heart Association task force on practice guidelines. Circulation. 2011;123(18):e426-579.

17. Mui AC. Geriatric depression scale as a community screening instrument for elderly Chinese immigrants. Int Psychogeriatr. 1996;8(3):445-58. 
18. Yesavage JA, Brink TL, Rose TL, Lum O, Huang V, Adey M, et al. Development and validation of a geriatric depression screening scale: a preliminary report. J Psychiatr Res. 1982;17(1):37-49.

19. Bassett DR Jr. International physical activity questionnaire: 12-country reliability and validity. Med Sci Sports Exerc. 2003;35(8):1396.

20. Kaiser MJ, Bauer JM, Ramsch C, Uter W, Guigoz Y, Cederholm T, et al. Validation of the Mini nutritional assessment short-form (MNA-SF): a practical tool for identification of nutritional status. J Nutr Health Aging. 2009;13(9):782-8.

21. Liguori I, Curcio F, Russo G, Cellurale M, Aran L, Bulli G, et al. Risk of malnutrition evaluated by Mini nutritional assessment and sarcopenia in noninstitutionalized elderly people. Nutr Clin Pract. 2018;33(6):879-86.

22. Liu H, Song B, Jin J, Liu Y, Wen X, Cheng S, et al. Length of stay, hospital costs and mortality associated with comorbidity according to the Charlson comorbidity index in immobile patients after ischemic stroke in China: a National Study. Int J Health Policy Manag. 2021.

23. Zhang W, Shen S, Wang W, Zhou C, Xu L, Qiu J, et al. Poor lower extremity function was associated with pre-diabetes and diabetes in older chinese people. PLoS One. 2014;9(12):e115883.

24. Kim TN, Choi KM. The implications of sarcopenia and sarcopenic obesity on cardiometabolic disease. J Cell Biochem. 2015;116(7):1171-8.

25. Pedersen BK, Febbraio MA. Muscles, exercise and obesity: skeletal muscle as a secretory organ. Nat Rev Endocrinol. 2012;8(8):457-65.

26. Stapelberg NJ, Neumann DL, Shum DH, McConnell H, Hamilton-Craig I. A topographical map of the causal network of mechanisms underlying the relationship between major depressive disorder and coronary heart disease. Aust N Z J Psychiatry. 2011;45(5):351-69.

27. Sanada K, Miyachi M, Tanimoto M, Yamamoto K, Murakami H, Okumura S, et al. A cross-sectional study of sarcopenia in Japanese men and women: reference values and association with cardiovascular risk factors. Eur J Appl Physiol. 2010;110(1):57-65.

28. Oerkild B, Frederiksen M, Hansen JF, Simonsen L, Skovgaard LT, Prescott E. Home-based cardiac rehabilitation is as effective as Centre-based cardiac rehabilitation among elderly with coronary heart disease: results from a randomised clinical trial. Age Ageing. 2011;40(1):78-85.

29. Lichtman JH, Froelicher ES, Blumenthal JA, Carney RM, Doering LV, Frasure-Smith N, et al. Depression as a risk factor for poor prognosis among patients with acute coronary syndrome: systematic review and recommendations: a scientific statement from the American Heart Association. Circulation. 2014;129(12):1350-69.

30. Cacciatore F, Amarelli C, Maiello C, Pratillo M, Tosini P, Mattucci I, et al. Effect of Sacubitril-valsartan in reducing depression in patients with advanced heart failure. J Affect Disord. 2020;272:132-7.

31. Atkins JL, Wannamathee SG. Sarcopenic obesity in ageing: cardiovascular outcomes and mortality. Br J Nutr. 2020;124(10):1102-13.

\section{Publisher's Note}

Springer Nature remains neutral with regard to jurisdictional claims in published maps and institutional affiliations.

Ready to submit your research? Choose BMC and benefit from:

- fast, convenient online submission

- thorough peer review by experienced researchers in your field

- rapid publication on acceptance

- support for research data, including large and complex data types

- gold Open Access which fosters wider collaboration and increased citations

- maximum visibility for your research: over $100 \mathrm{M}$ website views per year

At BMC, research is always in progress.

Learn more biomedcentral.com/submissions 